Scientific Journal of Hamadan Nursing \& Midwifery Faculty - ISSN 2008-2819

\title{
The Relationship Between General Health Status and Quality of Life of Parents and Quality of Life of Children with Cancer
}

\author{
Fatemeh Cheraghi $^{1}$, Akram Feizy Barnaji ${ }^{2}$ Leili Tapak ${ }^{3}$, Amir Sadeghi ${ }^{4 *}$
}

1. Associate Professor, Research Center for Chronic Disease (Home Care), Hamadan University of Medial Sciences, Hamadan, Iran

2. MSc Student of Pediatric Nursing, Student Research Committee, School of Nursing and Midwifery, Hamadan University of Medical Sciences, Hamadan, Iran

3. Assistant Professor, Department of Biostatistics and Epidemiology, School of Public Health, Hamadan University of Medical Sciences, Hamadan, Iran

4. Assistant Professor, Nursing Department, Hamadan University of Medical Sciences, Hamadan, Iran

\begin{tabular}{lr}
\hline \multicolumn{1}{c}{ Article Info } \\
\hline & \\
& \\
Received: & $2017 / 05 / 22$ \\
Accepted: & $2017 / 06 / 24$ \\
Published Online & $2017 / 07 / 17$
\end{tabular}

DOI: $10.30699 /$ sjhnmf.26.5.6

Original Article

Use your device to scan and read the article online

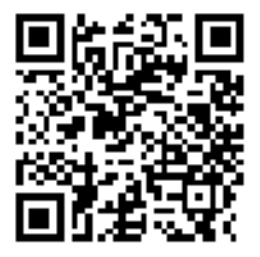

\section{Abstract}

Introduction: When the child has cancer, the family is faced with the challenge and this makes the parents spend a lot of energy caring for their children. In many cases it seems that the pressures of caring for sick children increases so much that affects the general health and quality of life of parents and causes malicious changes that affect children's quality of life in the end. This study aimed to determine the relationship between public health and quality of life of parents and quality of life of children with cancer at Besat Hospital, in 2016, respectively.

Methods: This was a correlation study, in which 108 mothers and fathers of children diagnosed with cancer with ages between 8-18 years old, referring to hematology department of Besat Clinic and hospital were selected by convenience sampling method. Data was gathered using general health questionnaires. The data was analyzed using SPSS20 and independent t-test and logistic regression analysis.

Results: The highest average scores in the aspects of quality of life for fathers were, physical functioning $(74 / 67 \pm 20 / 77)$, role impairment due to physical health $(61 / 20 \pm 28 / 25)$ and fatigue $(48 / 50 \pm 16 / 30)$ and for mothers were the pain scale $(50 / 97 \pm 23)$ and impairment role for emotional health (61/42 $\pm 31 / 94)$. There was just a significant relationship $(P=0 / 045)$ between anxiety symptoms and sleep disturbances, safety and quality of life of mothers of children. There was a significant relationship between the quality of life of mothers in terms of fatigue $(\mathrm{P}=0 / 011)$ and emotional well-being $(P=0 / 038)$ and fathers in children's pain and quality of life $(P=0 / 015)$.

Conclusion: Parents of children with cancer have problems in general health and quality of life. Health and social policies are required in order to plan properly to solve problems and improve the quality of life of parents and children.

Keywords: Cancer, Child, Quality of Life, Parents

Corresponding Information

Amir Sadeghi, Assistant Professor, Nursing Department, Hamadan University of Medical Sciences, Hamadan, Iran. Email: Ambehrad@yahoo.com Tel: 09181113956

Copyright (C) 2018, Sci J Hamadan Nurs Midwifery Fac. This is an open-access article distributed under the terms of the Creative Commons Attribution-noncommercial 4.0 International License which permits copy and redistribute the material just in noncommercial usages, provided the original work is properly cited.

How to Cite This Article:

Cheraghi F, Feizy Barnaji A, Sadeghi A, Tapak L. The Relationship Between General Health Status and Quality of Life of Parents and Quality of Life of Children with Cancer. Sci J Hamadan Nurs Midwifery Fac. 2018; 25 (5): 200-207 


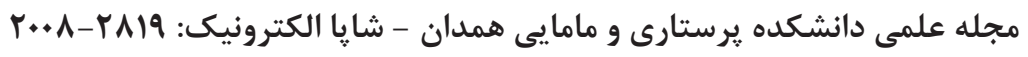

مقاله يثوهشى مأم همى

\title{
ارتباط سلامت عمومى و كيفيت زندكى والدين با كيفيت زندگى كودكان مبتلا به سرطان
}

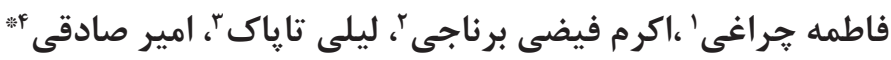 \\ • دانشيار، مركز تحقيقات مراقبت بيمارىهاى مزمن در منزل، دانشكدة برستارى و مامايى، دانشًاه علوم يزشكى همدان، همدان، \\ r. دانشجوى كارشناسى ارشد يرستارى كودكان، كميتأ تحقيقات دانشجويى، دانشكدة يرستارى و مامايى، دانشكاه علوم يزشكى

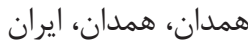

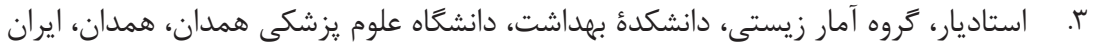

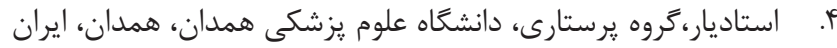

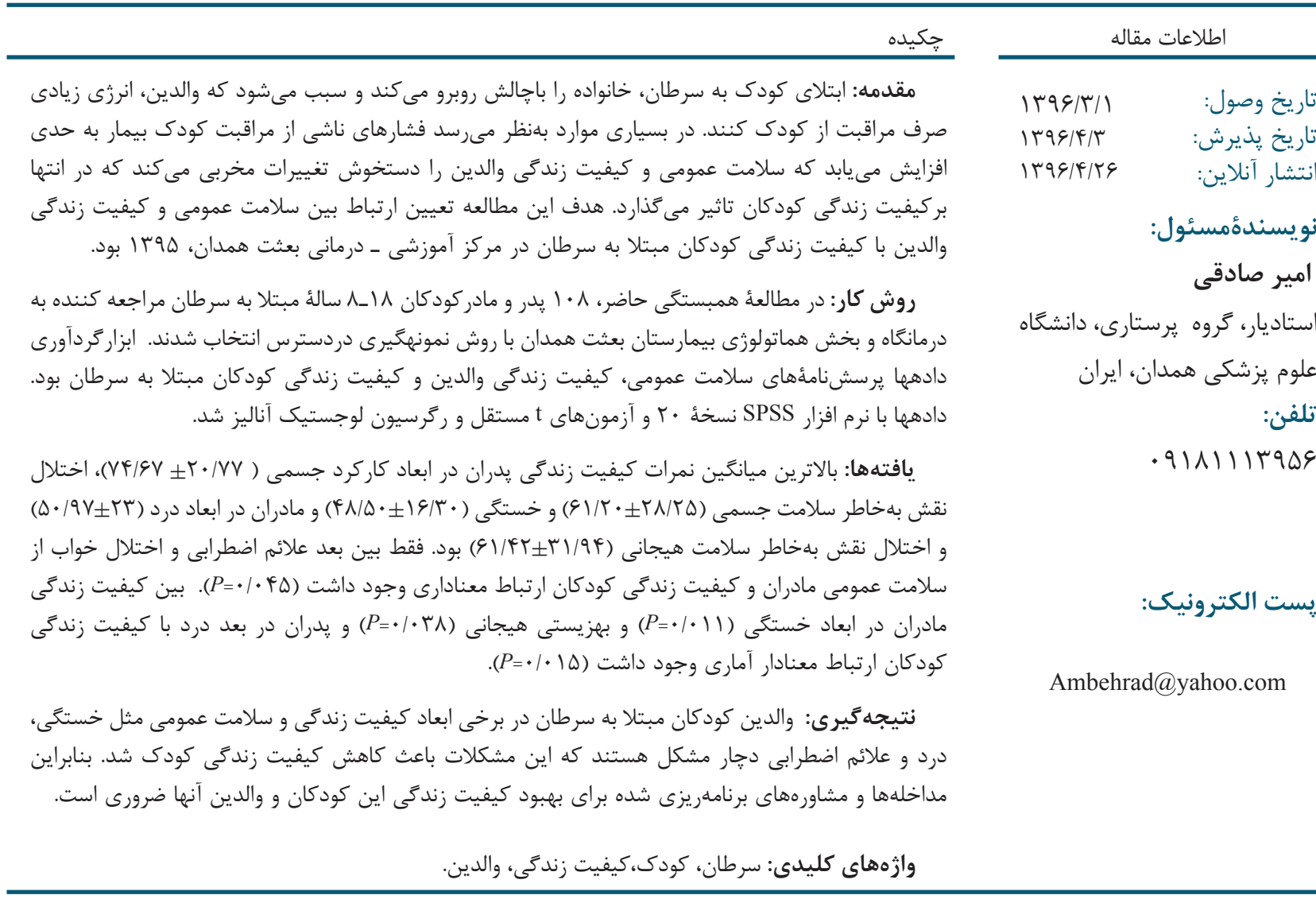

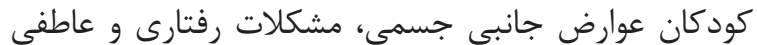

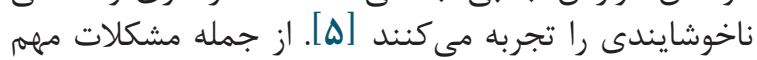

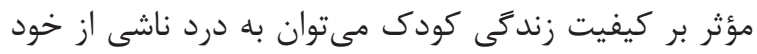

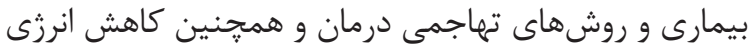

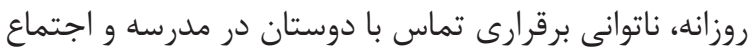

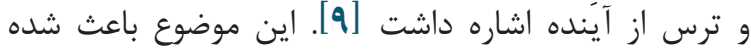

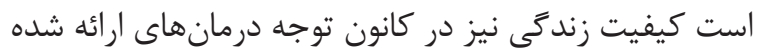

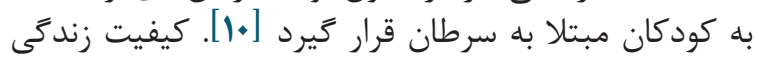

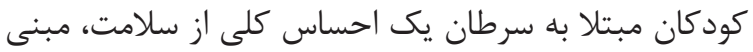

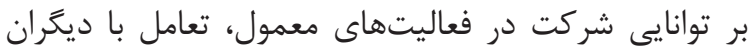

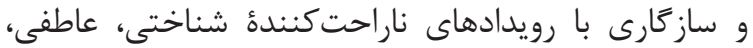

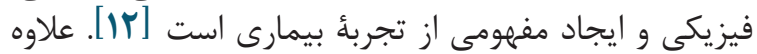

مقدمه

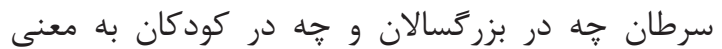
رشد غيرطبيعى سلول است [1]]. لوسمى و لنفور لنفوم شايعترين

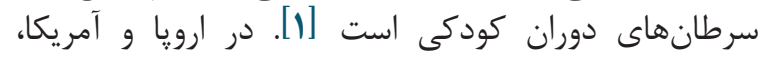

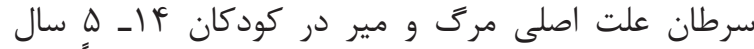

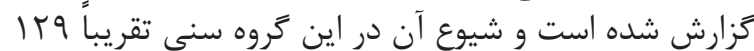

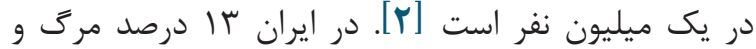

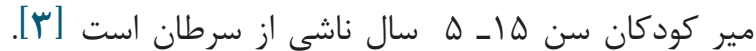

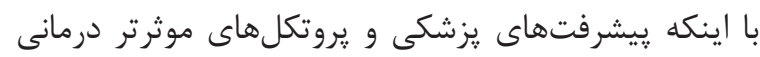

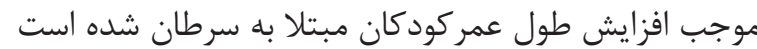

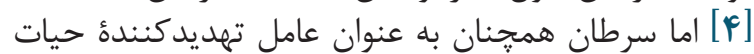

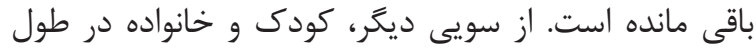

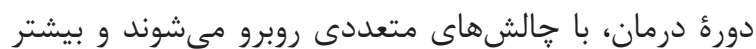




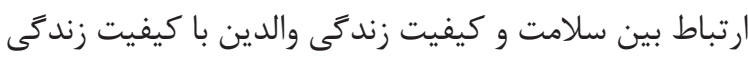

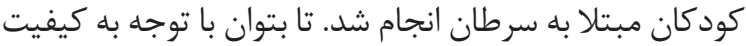

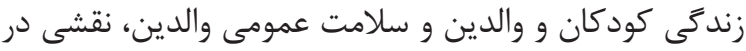

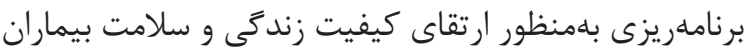

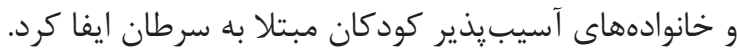

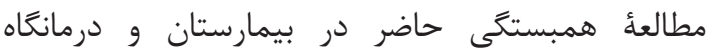

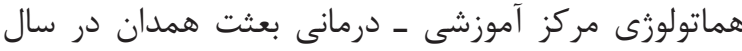

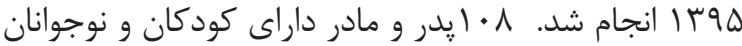

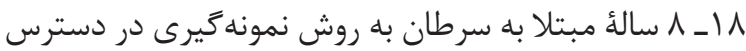

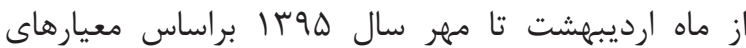

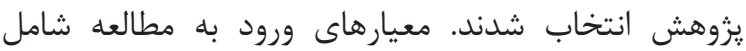

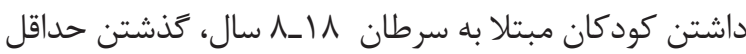

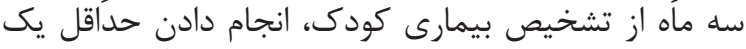

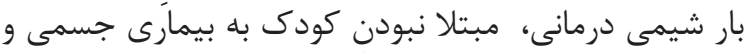

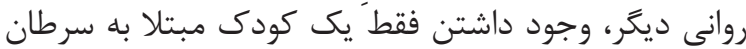

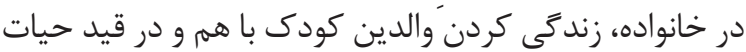

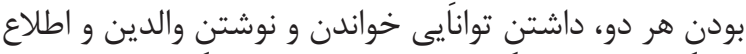

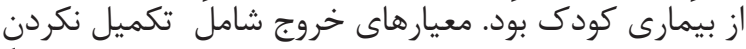

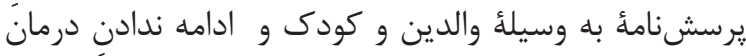
در مركز آموزشى درمانى بعثت بود

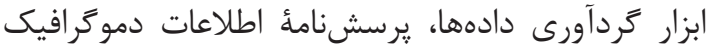

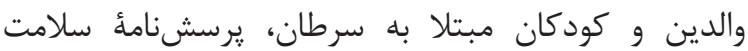

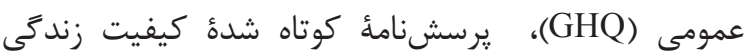

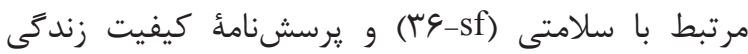
مرتبط با سلامت كودكان و نوجوانان كيندل (kindl) است كه مادر و يدر همأ يرسشناملهأها را تكميل كردند.

يرسشنامةٔ سلامت عمومى كلدبرك و هيلر در سال

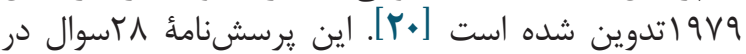

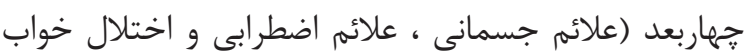

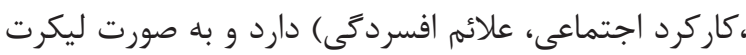

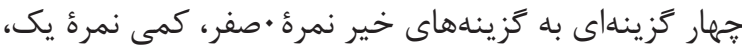

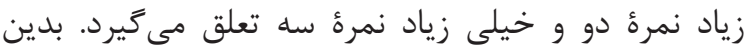

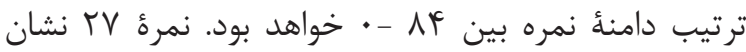

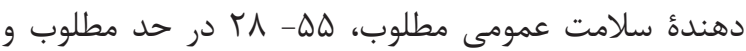

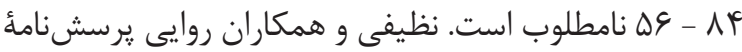

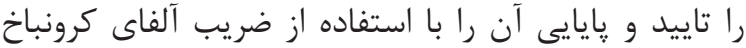

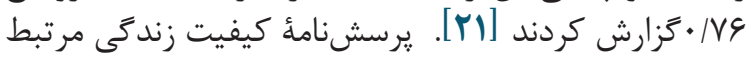

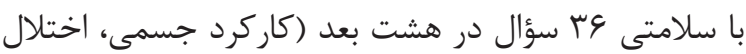

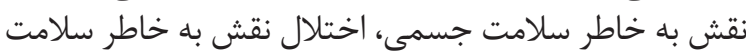

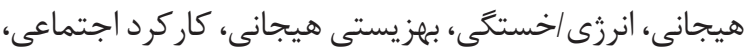

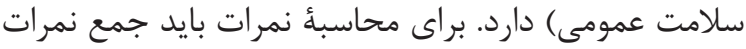

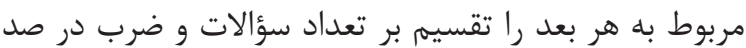

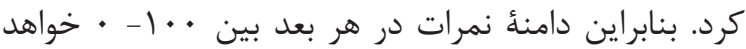

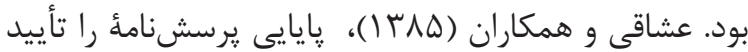

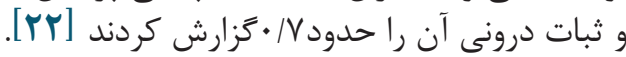

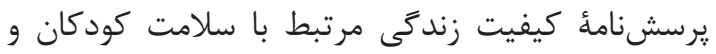

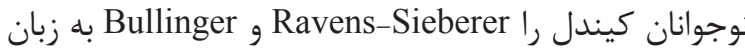
آلمانى در سال 1999 براى گروههاى سنى مختلف كودكان
بر آن سرطان عواقب كوتامددت و طولانىمدت نامطلوبى بر سلامت جسمى و روحى والدين دارد [عامدات طولانى

سرطان كودكان علاوه بر كودكان، بر والدين آنها نيز تاثير

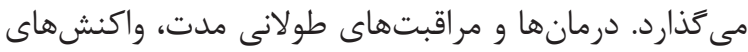

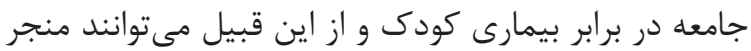

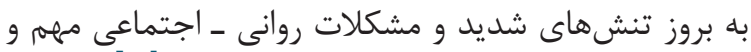

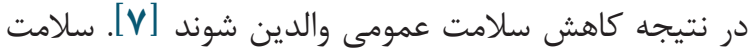

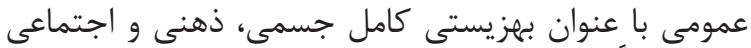

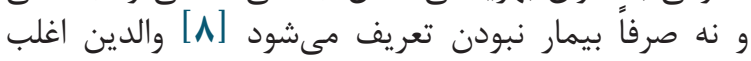

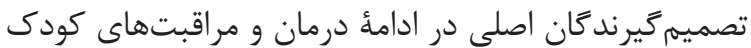

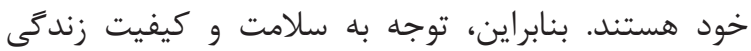

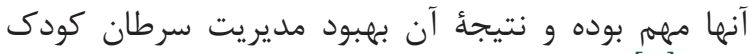

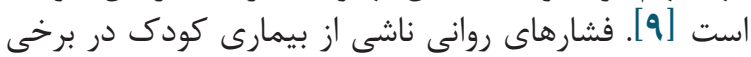

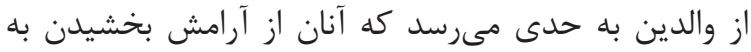

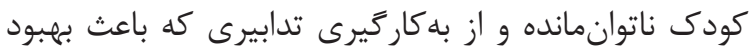

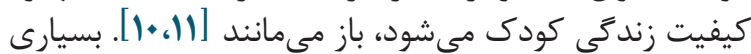

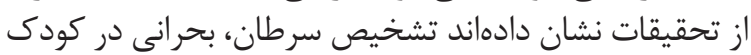

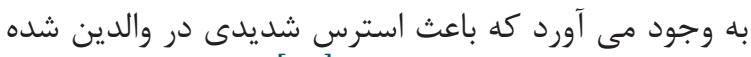

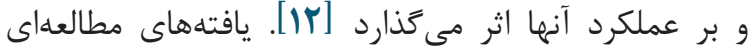

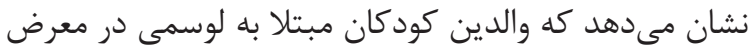

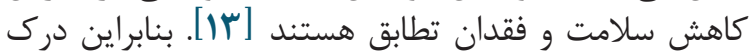

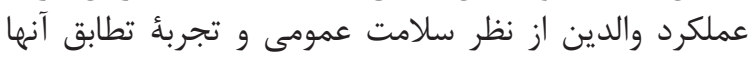

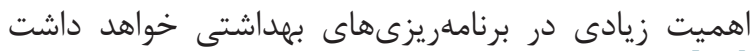

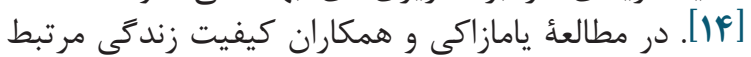

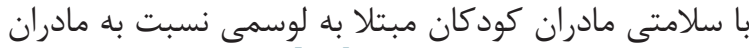

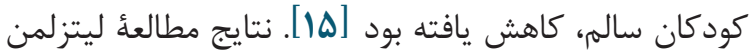

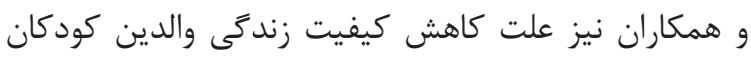

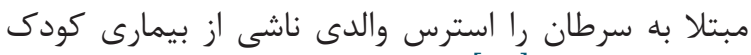

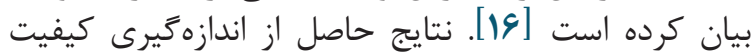

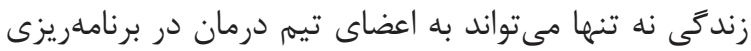

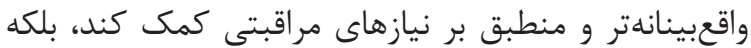

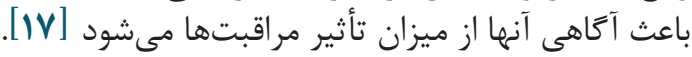

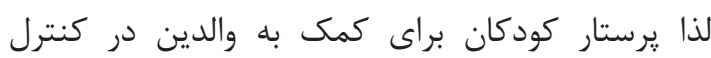

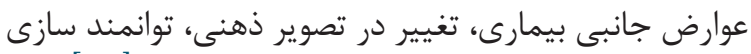

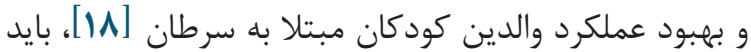

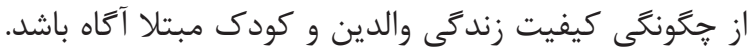

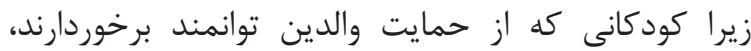

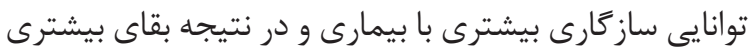

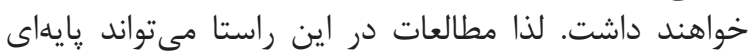

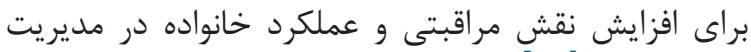

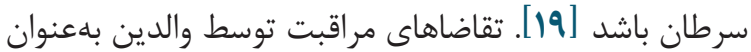

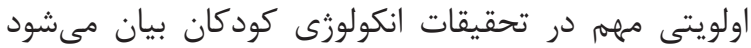

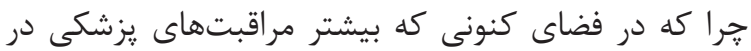

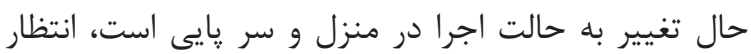

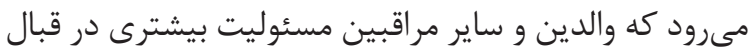

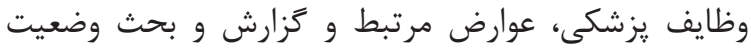

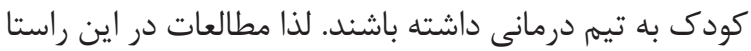

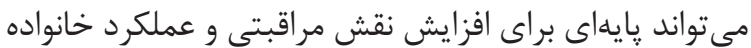

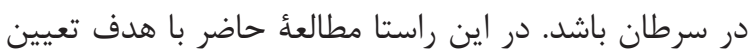




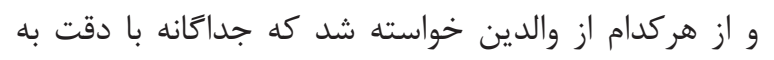

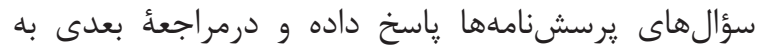

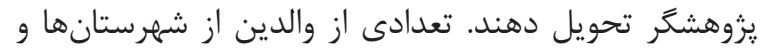

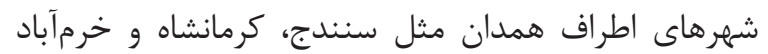

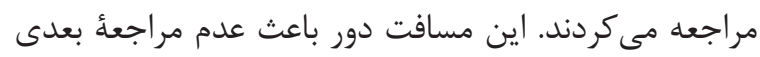

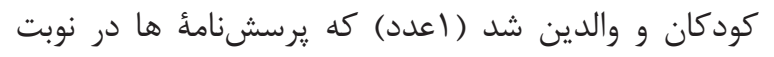

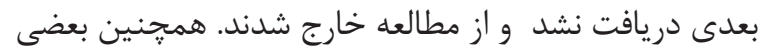

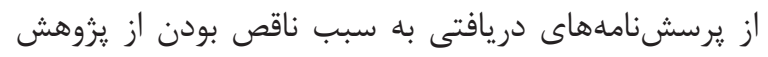

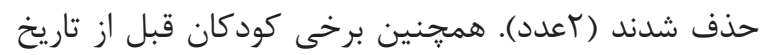

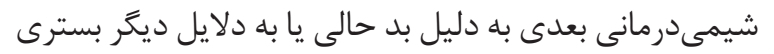

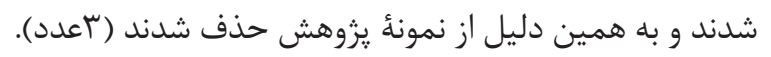

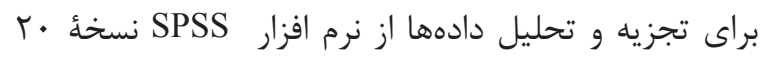

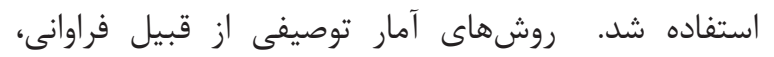

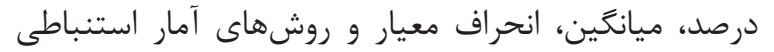

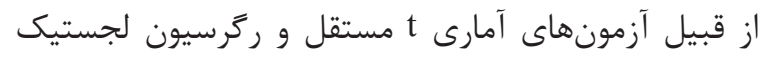

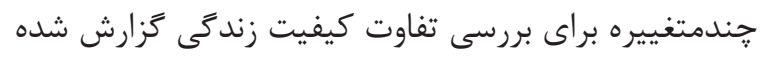

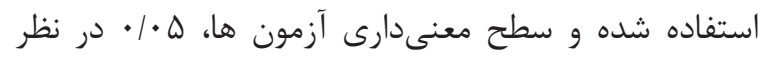
كرفته شد.

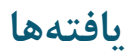

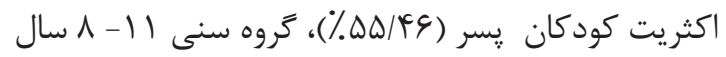

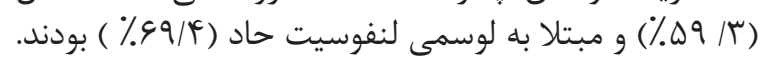

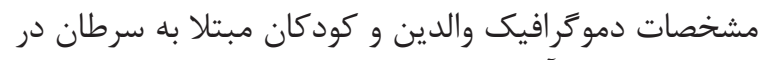
جدول شماره آ آمده است.

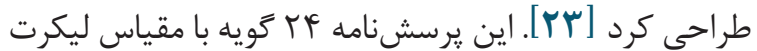

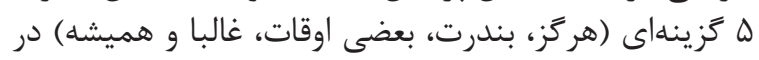

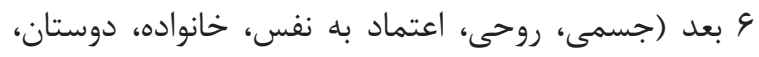

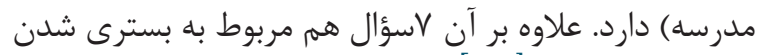

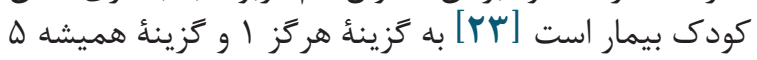

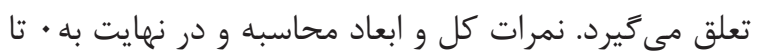

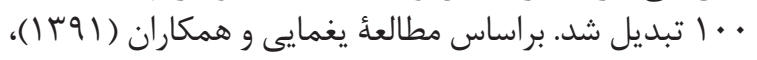

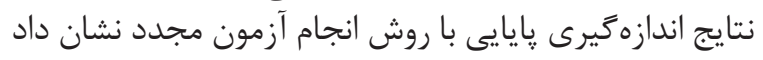

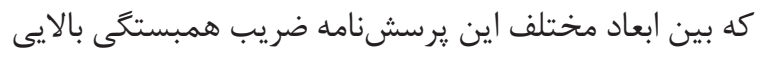

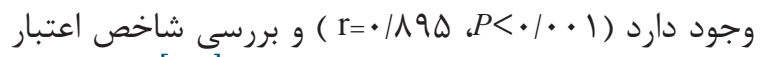

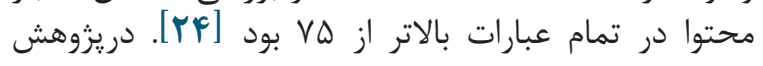

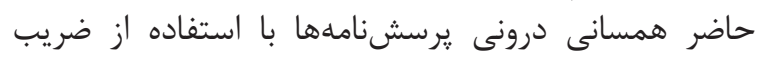

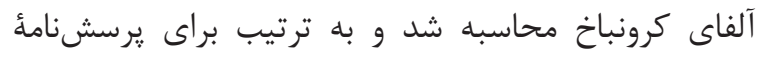

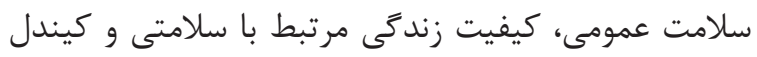

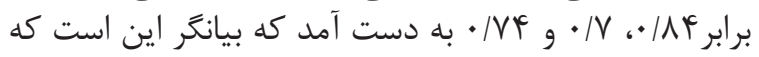

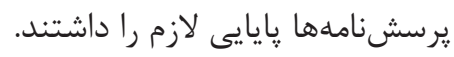

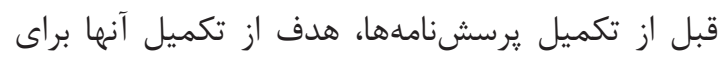

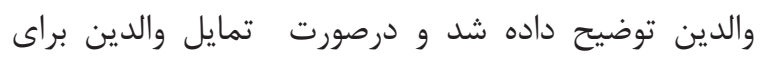

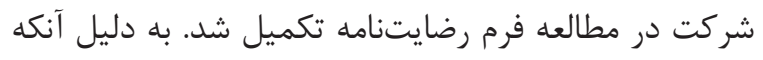

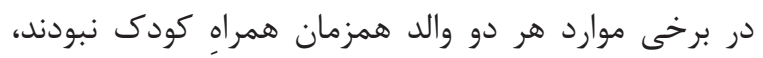

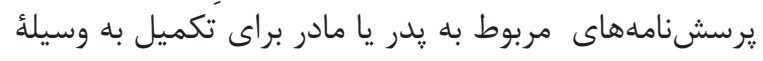

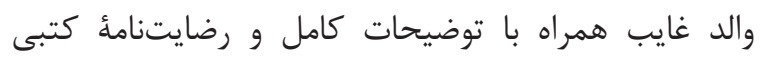

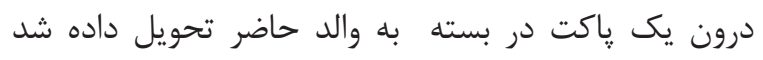

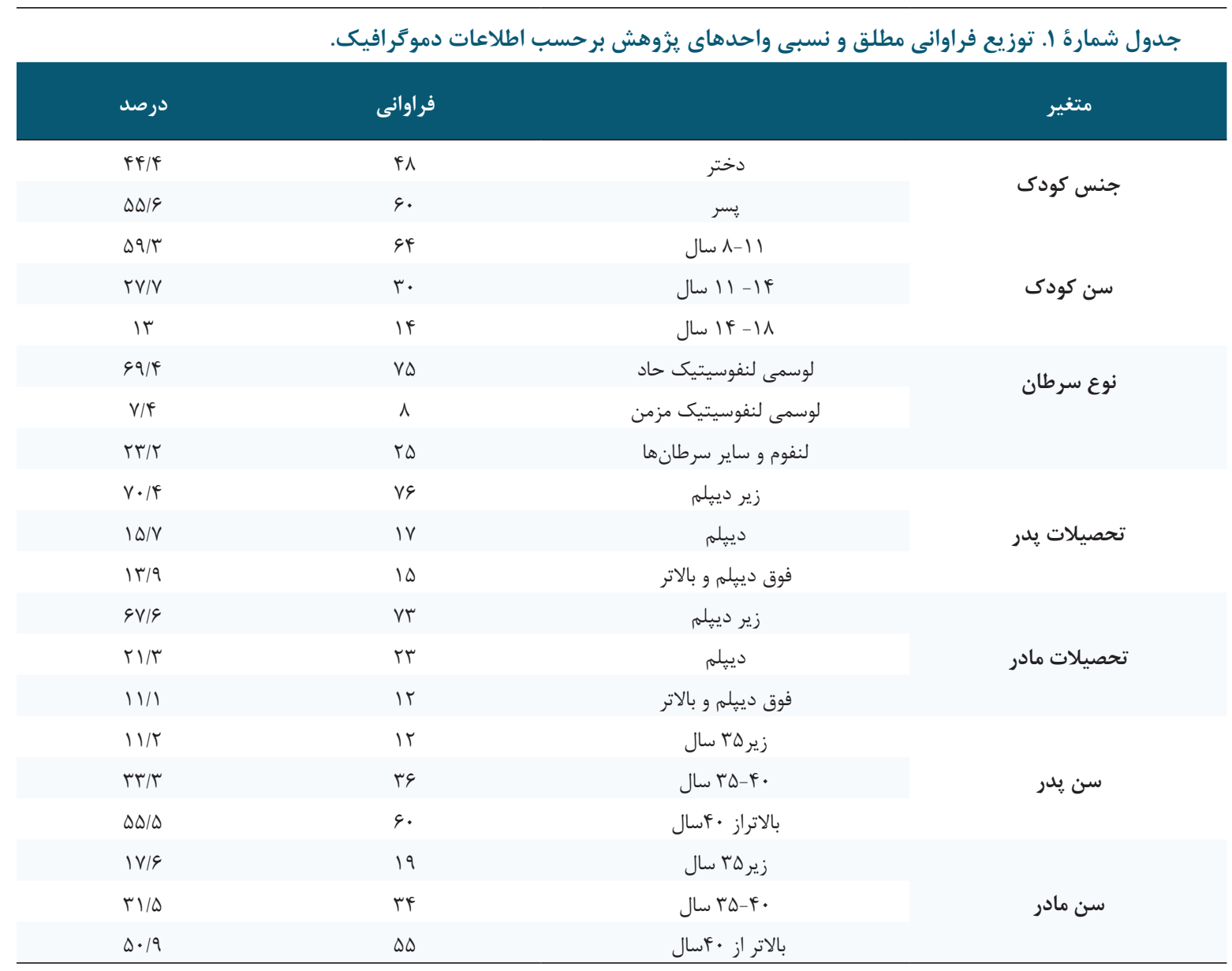




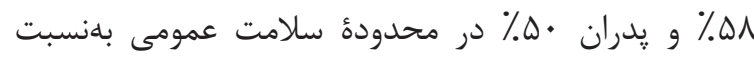

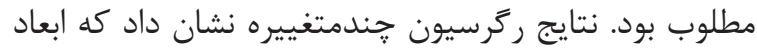

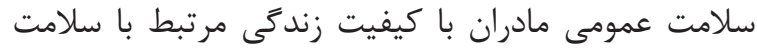

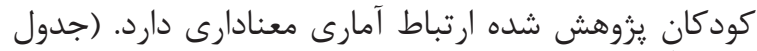

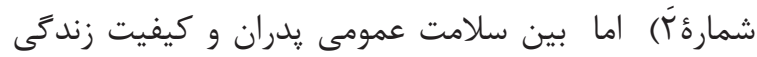

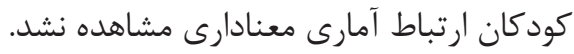

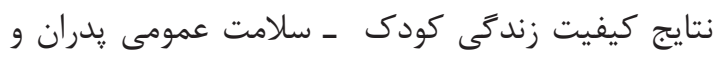

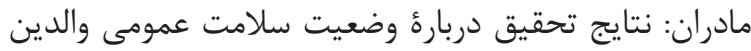

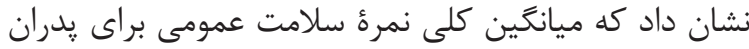

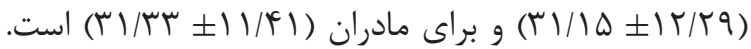

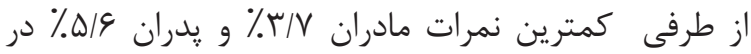

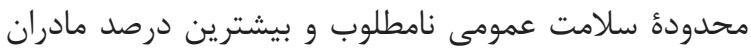

جدول شمارهُ ז. آزمون رَّرسيون لجستيك تعيين ارتباط سلامت عمومى يدر /مادر و كيفيت زندكى كودكان مطالعه شده.

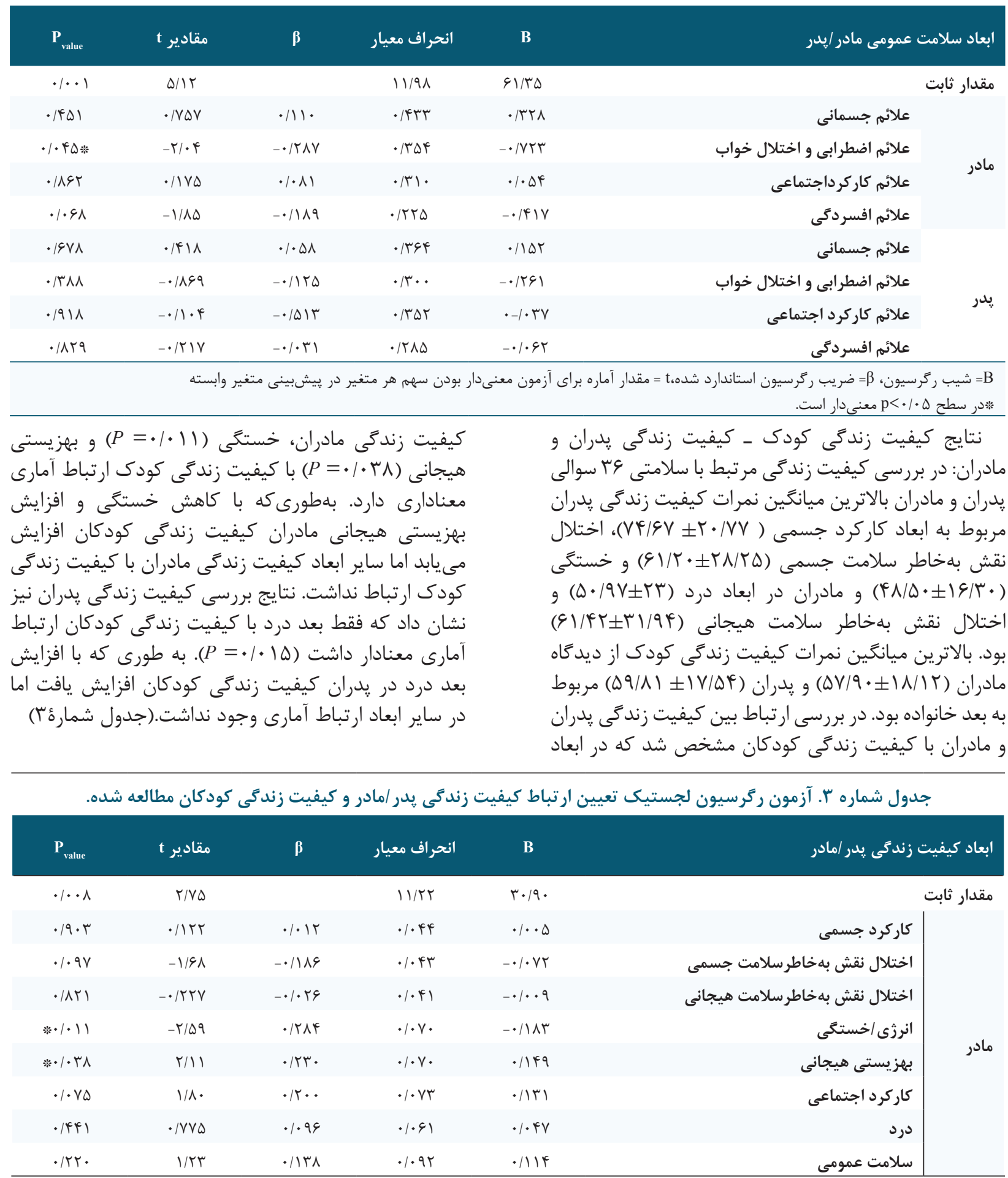




\begin{tabular}{|c|c|c|c|c|c|c|}
\hline $\mathbf{P}_{\text {value }}$ & مقادير t & $\boldsymbol{\beta}$ & انحراف معيار & B & \multicolumn{2}{|c|}{ ابعاد كيفيت زندكَى بدر /مادر } \\
\hline.$/ 9 \vee 1$ & ./.re & 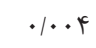 &.$\Delta r$ & $-\cdot / \cdot \cdot r$ & كاركرد جسمى & \multirow{8}{*}{ بدر } \\
\hline$\cdot / 199$ & $-\cdot / 1 r \mu$ & -.1 .19 & $.1 \cdot \mathrm{kV}$ & $-\cdot 1 \cdot \cdot 9$ & اختلال نقش بهخاطرسلامت جسمى & \\
\hline$\cdot /$ NTS & $-\cdot \pi k \cdot$ & $-\cdot / \cdot r V$ & $\cdot / \cdot{ }_{\mu}$ & $-\cdot / \cdot 14$ & اختلال نقش بهخاطرسلامت هيجانى & \\
\hline$\cdot 1099$ & $\cdot \operatorname{LSTA}$ & .1 .94 & $\cdot / \cdot \wedge$ &.$/ \cdot k t$ & انرثى اخستكى & \\
\hline$\cdot 1 \cdot \wedge$ & $1 / V V$ & . KAT &.$/ .9$ &.$/ 19$ & بهز يستى هيجانى & \\
\hline$\cdot 1011$ & $-\cdot \mid 991$ & -.1 .99 & .1 .94 & $-\cdot / \cdot k r$ & كاركرد اجتماعى & \\
\hline$* \cdot 1 \cdot 10$ & $r / r q$ & $\cdot / r \Delta V$ & $\cdot / \cdot \Delta T$ & $\cdot / 1 r$. & 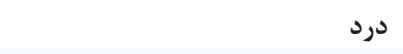 & \\
\hline$\cdot \pi r \Delta$ & $1 / Y r$ &.$/ 149$ & $\cdot / \cdot \wedge r$ & $\cdot 11 \cdot 1$ & سلامت عمومى & \\
\hline
\end{tabular}

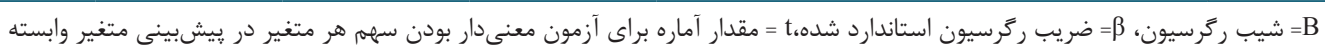

.

كزارش ضعيفترى از كيفيت زندكى مرتبط با سلامتى (به دردار

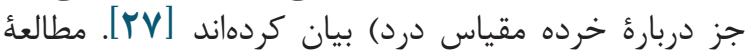
yamazaki

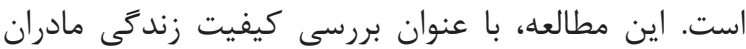

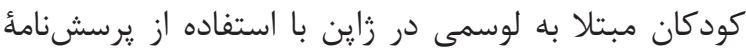

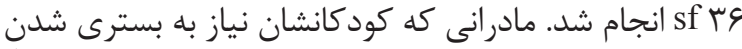

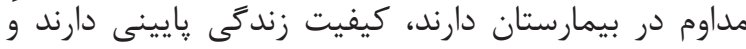

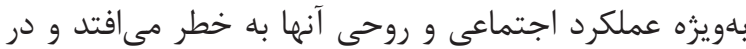

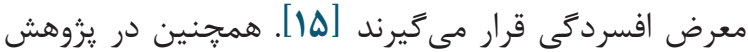

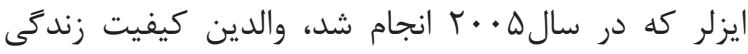

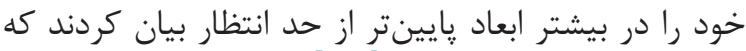

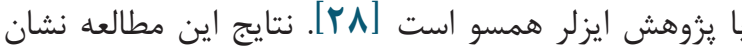

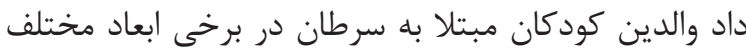

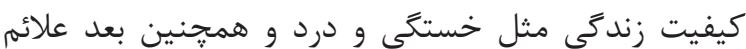

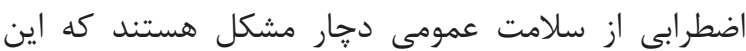

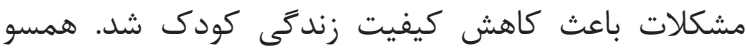

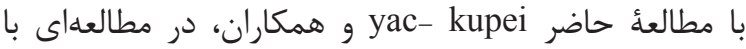

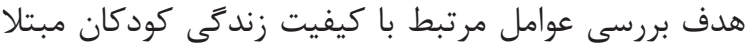

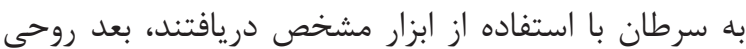

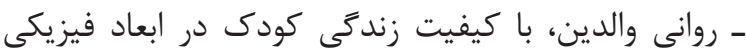

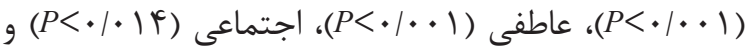

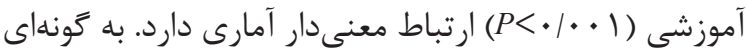

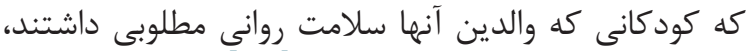

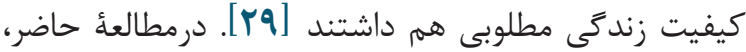

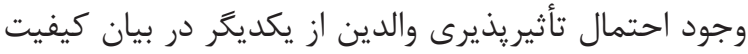

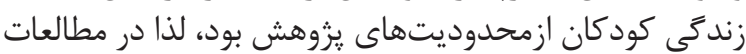

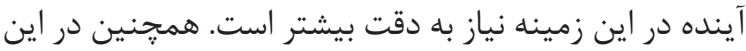

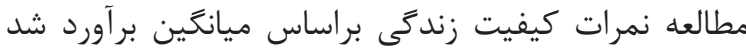

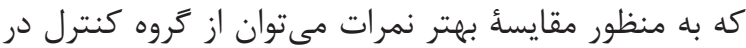
مطالعات آينده بهره برد.

\section{نتيجه}

با توجه به نتايج اين مطالعه، والدين كودكان و نوجوانان

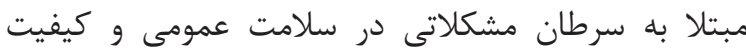

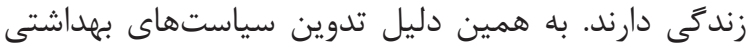

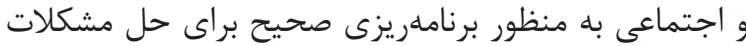
ابعاد مختلف كودكان و والدين آنها، انجام مداخلات مرات مناسب مثبات

نتايج اين مطالعه نشان داد كه سلامت عمومى نامطلوب

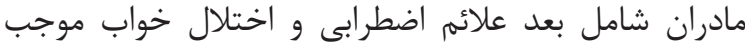

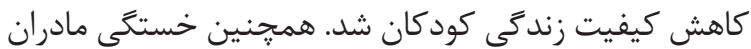

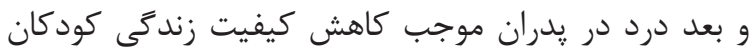

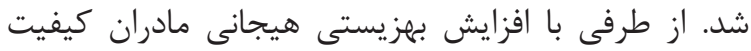

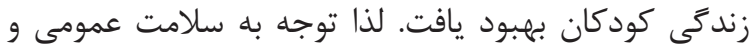

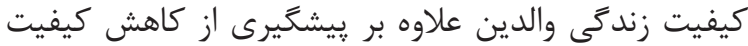

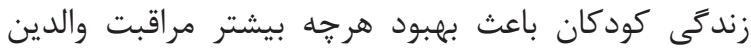

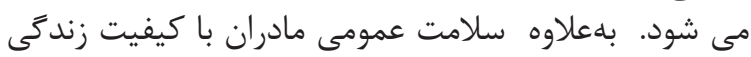

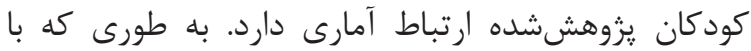

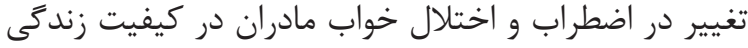

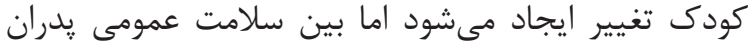

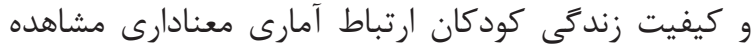

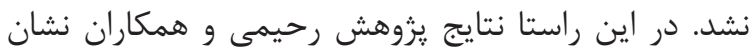

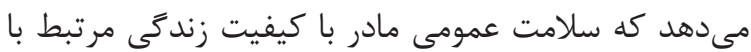

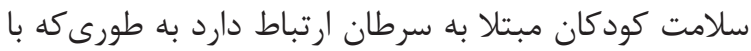

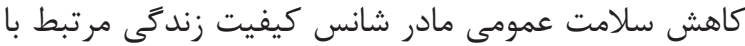

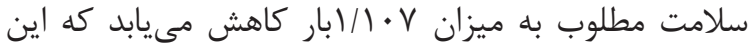

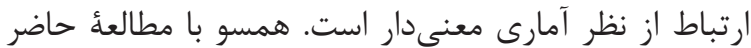

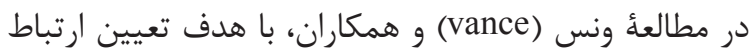

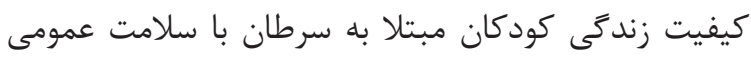

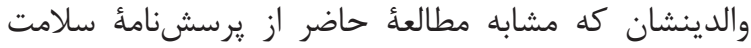

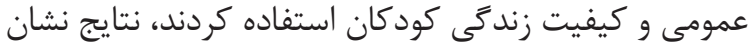

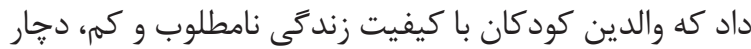

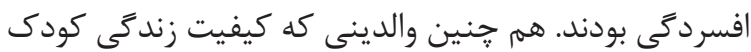

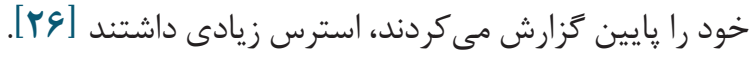
مطالعه حاضر نشان داد در بررسى كيفيت زند مئى يدران

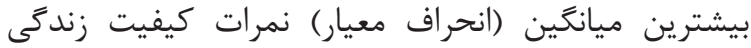

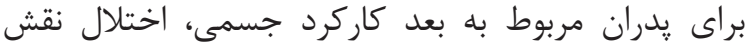

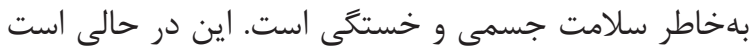

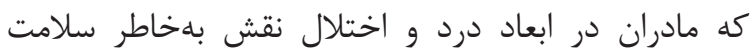

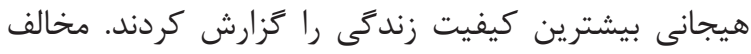

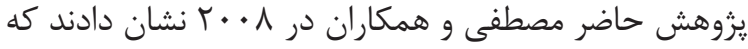

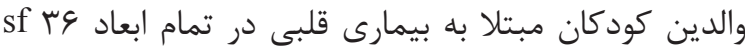

$$
\text { مجله علمى دانشكده يرستارى و مامايى همدان }
$$


با شماره طرح

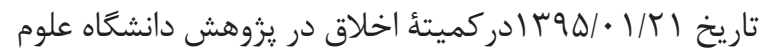

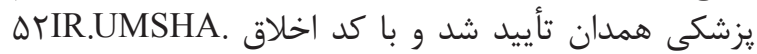

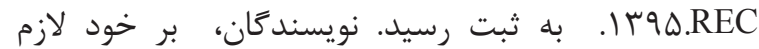

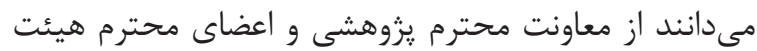

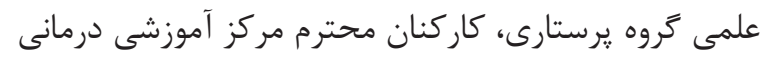

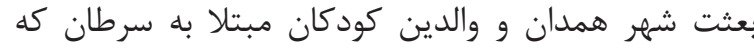
همكارى خود را دريغ نكردند، تشكر و قدردانى بنمايند.

$$
\text { تعارض در منافع }
$$

بين نويسند

$$
\begin{aligned}
& \text { براى كاهش عوارض جانبى درمانهاى شديد و تهاجمى إنى }
\end{aligned}
$$

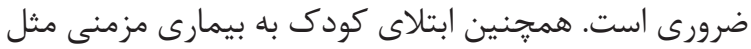

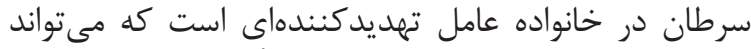

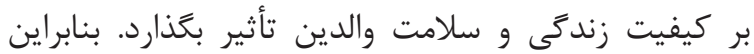

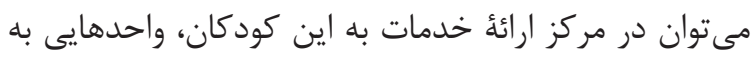

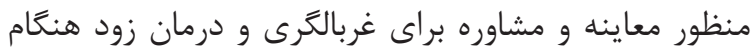

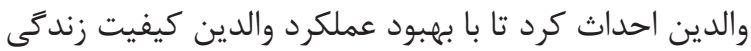

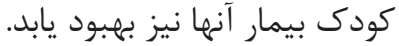

$$
\begin{aligned}
& \text { سياسَزارى }
\end{aligned}
$$

اين مقاله بركرفته از پايان نامئ دوره كارشناسى ارشد

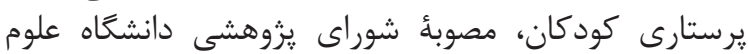

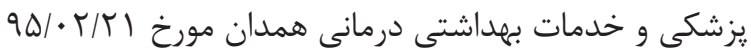

\section{References}

1. Karen G, markdent rm. Nelson Essentials of Pediatrics. 7nd ed. Tehran: Artin Teb; 2015.p.7845.

2. Wilson D, Hockenberry MJ. Wongs nursing care of infants and children. 8nd ed. Toronto: Elsevier; 2011.p.1536-80.

3. Mehranfar M, Younesi J, Banihashem A. Effectiveness of mindfulness-based cognitive therapy on reduction of depression and anxiety symptoms in mothers of children with cancer. Iran J Cancer Prev. 2012;5(1):1-7. PMid:25780532 PMCid:PMC4352519

4. Gatta G, Corazziari I, Magnani C, Peris-Bonet R, Roazzi P, Stiller C. Childhood cancer survival in Europe. Ann Oncol. 2003;14(suppl_5):v119-27.

5. Wallace H, Green D. Late Effects of Childhood Cancer. London: Arnold; 2004.

6. Grootenhuis MA, Last BF. Predictors of parental emotional adjustment to childhood cancer. Psychooncology. 1997;6(2):115-28. $\quad$ https://doi.org/10.1002/ (SICI) 1099-1611(199706)6:2<115::AIDPON252>3.0.CO;2-D

7. Hamed Tavasoli S, Alhani F. Evaluation of parent satiafactionn of nursing care in thalassemic children. J Urmia Nurs Midwifery Fac. 2011;9(1):9-11.

8. WHO. Guidelines for Drinking-water Quality Recommendations.: World Health Organization; 2004.

9. Barakat LP, Marmer PL, Schwartz LA. Quality of life of adolescents with cancer: family risks and resources. Health Qual Life Outcomes.

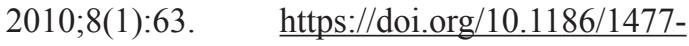
7525-8-63 PMid:20584303 PMCid:PMC2903513

10. Hinds PS, Billups CA, Cao X, Gattuso JS, Burghen E, West N, et al. Health-related quality of life in adolescents at the time of diagnosis with osteosarcoma or acute myeloid leukemia. Eur J Oncol Nurs. 2009;13(3):156-63. https://doi. org/10.1016/j.ejon.2008.08.003 PMid: 18926773 PMCid:PMC4017853

11. Hashemi F. How Do we Improve Quality of Life of Leukemic Children and their Families? Tehran: Barai Farda publication; 2011, p19-22.

12. Han HR. Korean mothers psychosocial adjustment to their children's cancer. J Adv Nurs. 2003;44(5):499-506. https://doi.org/10.1046/ j.0309-2402.2003.02833.x PMid: 14651698

13. Ozer ZC, Firat MZ, Bektas HA. Confirmatory and exploratory factor analysis of the caregiver quality of life index-cancer with Turkish samples. Qual Life Res. 2009;18(7):913-21. https://doi.org/10.1007/s11136-009-9503-1 PMid: 19554474

14. Wong MYF, Chan SWC. The qualitative experience of Chinese parents with children diagnosed of cancer. J Clin Nurs. 2006;15(6):710-7. https:// doi.org/10.1111/j.1365-2702.2006.01297.x PMid: 16684166

15. Yamazaki S, Sokejima S, Mizoue T, Eboshida A, Fukuhara S. Health-related quality of life of mothers of children with leukemia in Japan. Qual Life Res. 2005;14(4):1079-85. https://doi.org/10.1007/s11136-004-3288-z PMid: 16041903

16. Litzelman K, Catrine K, Gangnon R, Witt WP. Quality of life among parents of children with cancer or brain tumors: the impact of child characteristics and parental psychosocial factors. Qual Life Res. 2011;20(8):1261-9. https://doi. org/10.1007/s11136-011-9854-2 PMid:21287280 PMCid:PMC3121891

17. Potter PA, Perry AG. Fundamentals Of Nursing. 6nd ed. Uk: Mosby Co; 2004. 


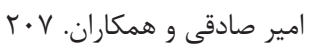

18. King CR, Hinds PS. Quality of life: from nursing and patient perspectives. Boston: Jones \& Bartlett; 2003.

19. Wells DK, James K, Stewart JL, Moore IMK, Kelly KP, Moore B, et al. The care of my child with cancer: A new instrument to measure caregiving demand in parents of children with cancer. J Pediatr Nurs. 2002;17(3):201-10. https://doi. org/10.1053/jpdn.2002.124113 PMid:12094361

20. Goldberg DP, Hillier VF. A scaled version of the General Health Questionnaire. Psychol Med. 1979;9(1):139-45. https://doi.org/10.1017/ $\underline{\mathrm{S} 0033291700021644}$

21. Nazifi M, Mokarami H, Akbaritabar A, Faraji Kujerdi M, Tabrizi R, Rahi A. Reliability, Validity and Factor Structure of the Persian Translation of General Health Questionnaire (GHQ-28) in Hospitals of Kerman University of Medical Sciences. J Fasa Univ Med Sci. 2014;3(4):336-42.

22. Eshaghi S-R, Ramezani MA, Shahsanaee A, Pooya A. Validity and reliability of the Short Form-36 Items questionnaire as a measure of quality of life in elderly Iranian population. Am J Appl Sci. 2006;3(3):1763-6. https://doi. org/10.3844/ajassp.2006.1763.1766

23. Ravens-Sieberer U, Bullinger M. Assessing health-related quality of life in chronically ill children with the German KINDL: first psychometric and content analytical results. Qual Life Res. 1998;7(5):399-407. https://doi. org/10.1023/A:1008853819715 PMid: $\underline{961720}$
24. Yaghmaee F, Aminzade S, Alavi Majd H, Rasoly M, Norian M. Translation and reviews psychological characteristics children health-related quality of life questionnaire kindle. Payesh. 2013;11(6):841-7.

25. Rahimi S, Fadakar Soghe K, Tabari R, Kazem Nejad Lili E. Relationship between Mother's General Health Status with Quality of Life of Child with Cancer. HAYAT. 2013;19(2).

26. Vance YH, Jenney ME, Eiser C, Morse RC. Issues in measuring quality of life in childhood cancer: measures, proxies, and parental mental health. J Child Psychol Psychiatry. 2001;42(5):6617.https://doi.org/10.1111/1469-7610.00761 PMid: 11464970

27. Arafa MA, Zaher SR, El-Dowaty AA, Moneeb DE. Quality of life among parents of children with heart disease. Health Qual Life Outcomes. 2008;6(1):91.https://doi.org/10.1186/1477-75256-91 PMid:18980676 PMCid:PMC2590598

28. Eiser C, Eiser JR, Stride CB. Quality of life in children newly diagnosed with cancer and their mothers. Health Qual Life Outcomes. 2005;3(1):29. https://doi.org/10.1186/1477-7525-3-29 PMid:15860126 PMCid:PMC1097748

29. Yagc-Küpeli B, Akyüz C, Küpeli S, Büyükpamukçu M. Health-related quality of life in pediatric cancer survivors: a multifactorial assessment including parental factors. J Pediatr Hematol Oncol. 2012;34(3):194-9. PMid:22441710 